\section{REVISTA BRASILEIRA DE QUALIDADE DE VIDA}

\title{
Qualidade de vida dos fisioterapeutas e colaboradores do setor de fisioterapia aquática da Associação de Assistência à Criança Deficiente (AACD)
}

\section{Quality of life profile of physiotherapists and staff of aquatic physiotherapy sector association of child care poor (AACD)}

\author{
Fernanda Simões Risco Bert \\ Associação de Assistência à Criança Deficiente - AACD - São Paulo - Brasil \\ fernandariscobert@gmail.com \\ Douglas Braga \\ Associação de Assistência à Criança Deficiente - AACD - São Paulo - Brasil \\ dbraga@aacd.org.br
}

\section{RESUMO}

OBJETIVO: Esta pesquisa teve como finalidade investigar a qualidade de vida dos fisioterapeutas aquáticos e colaboradores do setor de fisioterapia aquática da Associação de Assistência à Criança Deficiente (AACD).

MÉTODOS: Para a coleta de dados foi aplicado o questionário World Health Organization Quality of Life-100 (WHOQOL-100). A amostra da pesquisa contou com 29 fisioterapeutas aquáticos e colaboradores do setor de fisioterapia aquática da AACD, assim distribuídos: 24 fisioterapeutas aquáticos e 5 colaboradores. Para a análise das variáveis não paramétricas, utilizou-se o coeficiente de correlação linear de Spearman (r) através do programa Prism® version 4, sendo considerado como significante o valor de $\mathrm{p}$ inferior a 0,05 .

RESULTADOS: Foi observada correlação entre os Domínios ambiente e psicológico com a qualidade de vida. Dentro dos Domínios, as facetas pensar, aprender, memória e concentração e ambiente físico, respectivamente, foram as que mais interferiram nos Domínios.

CONCLUSÃO: A qualidade de vida dos fisioterapeutas e colaboradores que trabalham na fisioterapia aquática da AACD sofre alteração pelo ambiente físico e aspectos psicológicos.

PALAVRAS-CHAVE: Qualidade de vida. Trabalho. Fisioterapia. WHOQOL-100.

\section{ABSTRACT}

OBJECTIVE: The aim of this research was to investigate the quality of life of aquatic therapists and other employees who work at Association of Assistance to disabled child (AACD).

METHODS: For data collection was applied a World Health Organization Quality of Life (WHOQOL-100) questionnaire. The study sample consisted of 29 employees: 24 were aquatic therapists and 5 worked in other positions in AADC aquatic therapy service. For the analysis of nonparametric variables it was used Spearman linear correlation coefficient, and the Prism version 4 program. It was considered significant value when $\mathrm{p}$ was less than 0,05 . 
RESULTS: It was observed correlation between environment and psychological fields with quality of life. Within the Domains, the facets thinking, learning, memory, concentration, and physical environment, respectively, had the most influence in the Domains.

CONCLUSION: The quality of life of all employees working at AADC aquatic therapy service is influenced by physical environment and psychological aspects.

KEYWORDS: Quality of life Work. Physical Therapy. WHOQOL-100.

\section{Introdução}

A qualidade de vida no ambiente de trabalho do profissional da saúde é dependente de fatores físicos e mentais, podendo influenciar na qualidade do atendimento ao cliente. Estudiosos enfatizam a importância da saúde do trabalhador em seu ambiente como forma de prevenir o absenteísmo e o abandono de emprego (CARVALHO; MALAGRIS, 2007; MINAYO, 1994; MINAYO; HARTZ; BUSS, 2000).

$\mathrm{Na}$ área da saúde e das políticas públicas, o interesse pelo conceito de qualidade de vida no ambiente de trabalho é relativamente recente. A qualidade de vida é um indicador possível para a avaliação da eficácia, da eficiência e do impacto de fatores determinantes tanto no tratamento para grupos portadores de diversos agravos, quanto na comparação entre procedimentos para o controle de problemas ligados à saúde (SEIDL; ZANNON, 2004).

A qualidade de vida traz inúmeros benefícios para o bem-estar das pessoas e uma maior produtividade (CANIZARES, 2008), bem como, influi diretamente na execução das metas de uma organização. A empresa que não atenta para a qualidade de vida de seus funcionários pode se considerar decadente, uma vez que seus clientes internos não estão satisfeitos, acabam refletindo esta insatisfação na sua produtividade (LACAZ, 2000).

As empresas que oferecem serviços de saúde estão cada vez mais atentas à qualidade de vida de seus profissionais, oferecendo ações interligadas que abrangem melhorias, inovações gerenciais, tecnológicas e estruturais no ambiente e preparando o trabalhador para o cumprimento de um novo ciclo de trabalho (CANIZARES, 2008).

O fisioterapeuta é um profissional de nível superior da área de saúde, pleno, autônomo, com atuação isolada ou em equipe, em todos os níveis de assistência à saúde, incluindo prevenção, promoção, desenvolvimento, tratamento e recuperação da saúde em indivíduos, grupos de pessoas ou comunidade (BARROS, 2008). É o profissional da saúde que se ocupa do movimento humano em toda a sua plenitude, com o objetivo de preservar, manter, desenvolver e restaurar a integridade de órgãos, sistemas e funções corporais (BATISTA, 2010). Esse profissional emprega conhecimentos científicos e lança mão de técnicas e recursos fisioterápicos manuais e de agentes físicos como eletroterapia, hidroterapia, fototerapia, termoterapia, equoterapia e cinesioterapia, na prevenção, no tratamento e na reabilitação das condições de vida e de saúde do indivíduo (BARROS, 2008; BATISTA, 2010).

A hidroterapia é mais um dos recursos utilizados pelo profissional fisioterapeuta. Atualmente, o termo mais aceito é fisioterapia aquática, que é a terapia de reabilitação física utilizando exercícios, manuseios e técnicas específicas associadas às propriedades do meio líquido, com o objetivo de promover ganhos específicos que possam ser transferidos para o solo e, portanto, traduzidos em ganhos funcionais (SILVA; BRANCO, 2011).

O fisioterapeuta que trabalha no setor de hidroterapia depende das condições do ambiente físico para exercer sua função, e estas condições são peculiares. Destaca-se as condições térmicas relacionadas à temperatura da água da piscina e do ambiente, à acústica, ao lumínico. Essas condições, quando não adequadas, podem influenciar no atendimento do cliente e, também, na qualidade de vida do fisioterapeuta (SILVA; BRANCO, 2011; SKINNER; THOMSON, 1985). 
Pensando no ambiente de trabalho, o intuito dessa pesquisa é investigar a qualidade de vida dos fisioterapeutas e colaboradores do setor de fisioterapia aquática da Associação de Assistência à Criança Deficiente (AACD).

\section{Métodos}

Trata-se de um estudo transversal, descritivo e quantitativo desenvolvido no setor de fisioterapia aquática da AACD.

Para a efetivação deste trabalho foi aplicado o questionário World Health Organization Quality of Life-100 (WHOQOL-100) no período de janeiro a fevereiro de 2013.

O WHOQOL-100, desenvolvido pela Organização Mundial da Saúde, contém 100 perguntas distribuídas em 6 Domínios: físico, psicológico, nível de independência, relações sociais, meio ambiente e espiritualidade/religiosidade/crenças pessoais. Esses Domínios são divididos em 24 facetas (FLECK, 2000) e cada faceta é composta por 4 perguntas. Além das 24 facetas específicas, o instrumento tem uma faceta adicional que não está inserida nos Domínios, composta de quatro perguntas gerais sobre qualidade de vida.

Após a coleta dos dados, foram realizados tratamentos estatísticos, correlacionando-se os Domínios com as respectivas facetas e também cada Domínio com o respectivo valor total da escala utilizada (FLECK, 2000).

Para as variáveis não paramétricas (Domínio/faceta) utilizou-se o coeficiente de correlação linear de Spearman (r), com valores superiores a 0,8 configurando uma alta correlação, abaixo de 0,4 configurando uma baixa correlação e valores intermediários a esses foram considerados correlação moderada. Para a realização das análises estatísticas foi utilizado o programa Prism ${ }^{\circledR}$ version 4 , entendendo como significante o valor de p inferior a 0,05 .

O trabalho seguiu as recomendações da Resolução n 196/96, do Conselho Nacional de Saúde e foi submetido à avaliação da Plataforma Brasil, registro 20304413.6.0000.0085.

\section{Resultados}

A amostra foi composta por 24 fisioterapeutas e 5 colaboradores, sendo 10 do sexo masculino,

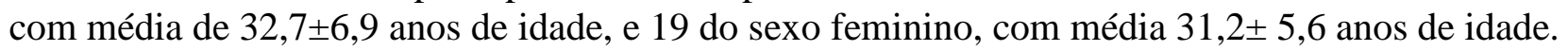

Os dados apresentados na Tabela 1 demonstram que os Domínios ambiente e psicológico foram os que tiveram maior correlação com o valor total da escala. O Domínio que teve menor correlação com a qualidade de vida foi o físico.

Tabela 1 - Valores das correlações entre cada Domínio avaliado e o valor total da escala WHOQOL-100

\begin{tabular}{lcc}
\hline \multicolumn{1}{c}{ Domínios Avaliados } & $\mathbf{r}$ & $\mathbf{p}$ \\
\hline Físico & 0,0781 & 0,6872 \\
Psicológico & $0,8301 \#$ & $<0,0001^{*}$ \\
Independência & 0,4941 & $0,0064^{*}$ \\
Relações Sociais & 0,4928 & $0,0066^{*}$ \\
Ambiente & $0,9139 \#$ & $<0,0001^{*}$ \\
Crenças & 0,4018 & $0,0307^{*}$ \\
\hline
\end{tabular}

Nota: $*=\mathrm{p}<0,05 ; \#=\mathrm{r}>0,8$.

Fonte: Autoria própria (2013).

No Domínio Físico (Tabela 2) pode-se observar que todas as facetas influenciam no resultado total do Domínio, porém somente a faceta dor e desconforto apresentou correlação com esse Domínio. Já no Domínio psicológico, das cinco facetas avaliadas, quatro facetas influenciaram no resultado total do Domínio, porém a faceta pensar, aprender, memória e concentração obteve correlação com o Domínio psicológico.

No Domínio Nível de Independência e suas respectivas facetas (Tabela 2), observou-se que nenhuma das facetas apresentou correlação com o Domínio. Somente ocorre correlação moderada na 
faceta mobilidade e correlação baixa nas facetas atividades de vida cotidiana, capacidade de trabalho e dependência de medicação ou tratamentos.

No Domínio Relações Sociais (Tabela 2) todas as facetas influenciaram na qualidade de vida dos fisioterapeutas e colaboradores do setor de fisioterapia aquática, porém, somente a faceta suporte social apresentou correlação no Domínio relações sociais.

No Domínio Ambiente (Tabela 2) ocorreu influência de seis das oito facetas avaliadas, porém, a faceta que apresentou coeficiente de correlação foi ambiente físico. O Domínio aspectos espirituais/religião/crenças não é composto por nenhuma faceta, sendo assim, comparado com os outros Domínios, não influencia na qualidade de vida da amostra avaliada.

Tabela 2 - Correlação dos Domínios e suas respectivas facetas

\begin{tabular}{|c|c|c|}
\hline Domínio /Faceta & $\mathbf{r}$ & $\mathbf{p}$ \\
\hline \multicolumn{3}{|l|}{ Domínio Físico/Faceta } \\
\hline Dor e desconforto & $0,8356 \#$ & $0,0001 *$ \\
\hline Energia e fadiga & 0,4958 & $0,0063 *$ \\
\hline Sono e repouso & 0,4573 & $0,0126^{*}$ \\
\hline \multicolumn{3}{|l|}{ Psicológico/Faceta } \\
\hline Sentimentos positivos & 0,6321 & $0,0002 *$ \\
\hline Pensar, aprender, memória e concentração & $0,8061 \#$ & $0,0001^{*}$ \\
\hline Autoestima & 0,7554 & $0,0001 *$ \\
\hline Imagem corporal e aparência & 0,5533 & $0,0018^{*}$ \\
\hline Sentimentos negativos & 0,1697 & 0,3788 \\
\hline \multicolumn{3}{|l|}{ Independência/Faceta } \\
\hline Mobilidade & 0,6017 & $0,0006^{*}$ \\
\hline Atividades de vida cotidiana & 0,4588 & $0,0123^{*}$ \\
\hline Dependência de medicação ou tratamentos & 0,2544 & 0,1835 \\
\hline Capacidade de trabalho & 0,4806 & $0,0083^{*}$ \\
\hline \multicolumn{3}{|l|}{ Relações Sociais } \\
\hline Relações sociais & 0,4939 & $0,0064 *$ \\
\hline Suporte social & $0,8269 \#$ & $0,0001 *$ \\
\hline Atividade sexual & 0,5458 & $0,0022 *$ \\
\hline \multicolumn{3}{|l|}{ Ambiente/Faceta } \\
\hline Segurança & 0,5365 & $0,0027 *$ \\
\hline Ambiente no lar & 0,5518 & $0,0019 *$ \\
\hline Recursos financeiros & 0,1585 & 0,4115 \\
\hline Cuidados de saúde e sociais & 0,5827 & $0,0009^{*}$ \\
\hline Oportunidade de adquirir novas informações e habilidades & 0,6696 & $0,0001^{*}$ \\
\hline Oportunidades de lazer & 0,6652 & $0,0001 *$ \\
\hline Ambiente físico & $0,8714 \#$ & $0,0001 *$ \\
\hline Transporte & 0,1239 & 0,5221 \\
\hline
\end{tabular}

Nota: $*=\mathrm{p}<0,05 ; \#=\mathrm{r}>0,8$

Fonte: Autoria própria (2013).

No Gráfico 1 nota-se a curva de correlação do Domínio Ambiente que apresentou uma maior correlação com os valores totais do WHOQOL-100.

Gráfico 1 - Correlação do Domínio Ambiente e WHOQOL-100

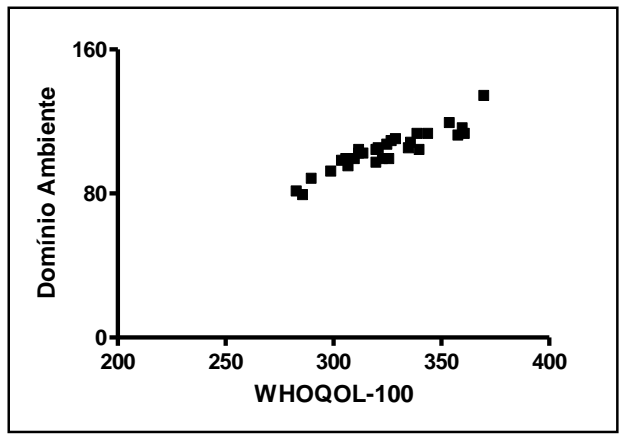

Nota: $*=\mathrm{p}<0,0001 ; \mathrm{r}=0,9139$.

Fonte: Autoria própria (2013). 


\section{Discussão}

Pode-se observar que dentre os seis Domínios avaliados na pesquisa, os Domínios que apresentaram coeficiente de correlação significativo com a qualidade de vida foram Domínio psicológico e Domínio ambiente.

No Domínio psicológico, a faceta que apresentou coeficiente de correlação alto foi pensar, aprender, memória e concentração. Esta faceta está diretamente relacionada ao aspecto psicológico dos fisioterapeutas e colaboradores. Sabe-se que a saúde emocional desta população está relacionada à adequada capacidade de gerenciamento de sentimentos, autoestima e entusiasmo em relação à vida, e que a utilização da capacidade criativa está ligada diretamente à capacidade de pensar, de aprender, de expandir conhecimentos permanentemente e de partilhar o potencial interno com os outros profissionais (RIBEIRO, 2001).

A relação entre paciente e terapeuta pode influenciar no aspecto psicológico dos profissionais da saúde. Ajudar outras pessoas sempre foi reconhecido como objetivo nobre, porém recentemente tem sido dada atenção para os custos emocionais da realização desse objetivo. O exercício desta profissão implica em uma relação com o cliente permeada de ambiguidades, como conviver com a tênue distinção entre se envolver profissionalmente e não pessoalmente na ajuda ao outro (CARVALHO; MALAGRIS, 2007; BENEVIDES-PEREIRA, 2002; NUNES; NASSARALLA, 2004). Acredita-se que, apesar deste estudo não abordar a relação paciente e terapeuta, esses profissionais dependem da relação para a evolução dos pacientes, podendo assim refletir em seu aspecto psicológico.

Outro fator inerente ao aspecto psicológico dos fisioterapeutas e funcionários é a busca pela melhora funcional dos pacientes, pois nesse trabalho os profissionais estão diante de uma causa onde o fator expectativa é cobrado em todos os momentos (BENEVIDES-PEREIRA, 2002).

Geralmente, o público atendido é composto por pessoas muito carentes ou que necessitam de cuidados extremos e intensivos por um tempo prolongado. Além desses fatores, não se pode esquecer da carga emocional de reabilitar pacientes com lesões neurológicas, muitas vezes crônicas e progressivas (BENEVIDES-PEREIRA, 2002; AMORIM, 2002; BATISTA 2010). Esse perfil é encontrado nos pacientes atendidos pelo centro de reabilitação da AACD.

A faceta suporte social apresentou correlação no Domínio Relações Sociais. Esse fato não é somente dependente da relação terapeuta e paciente, abrange o trabalho da equipe interdisciplinar. Essa equipe, além dos fisioterapeutas, é composta pelos funcionários do setor. São os funcionários do setor que, muitas vezes, realizam o primeiro contato com o paciente, sendo questionados sobre o funcionamento e dinâmica neste cenário de trabalho em equipe. É necessária a comunicação constante, e esta poderá influenciar no aspecto emocional destes funcionários. Esse aspecto é resultante de uma rede de significados que são constituídos por elementos cognitivos, afetivos e experiências, aglutinados nas relações sociais e culturais (COSTA, 2007, COSTA; ENDERS; MENEZES, 2008, ARAÚJO, 2009).

As exigências do trabalho devem corresponder às capacidades físicas e cognitivas do trabalhador. Quando há desequilíbrio entre estes dois fatores, há perdas tanto na qualidade do trabalho como na saúde do trabalhador. Um dos efeitos desse desequilíbrio é o estresse no trabalho (MAIA, 2007).

Neste estudo, das oito facetas analisadas, seis interferiram, mas somente uma apresentou coeficiente de correlação, que foi a faceta ambiente físico. Esse fato já era esperado, pois é a única variável comum na amostra estudada. A produtividade é dependente de elementos associados, como a interação ao ambiente de trabalho e a sensação de conforto ambiental. Em termos práticos, o ambiente físico responde às necessidades tanto em termos funcionais (físicos/cognitivos) quanto formais (psicológicos), tendo impacto positivo na realização das atividades de trabalho (VILLAROUCO; ANDRETO, 2008). 
Os indivíduos que compõem a amostra do presente estudo trabalham em condições especiais, em piscina terapêutica, que depende de diversos fatores, entre eles, o conforto térmico (temperatura da água e do ambiente) bem como o lumínico e o acústico.

No ambiente térmico existem duas condições: a temperatura do ambiente e a temperatura da água. Esses são fatores que podem influenciar o desempenho do fisioterapeuta e funcionários, pois, sabe-se que indivíduos que trabalham em ambientes quentes enfrentam desafios fisiológicos que podem comprometer o desenvolvimento de suas atividades profissionais (CAMARGO; FURLAN, 2011). Camargo e Furlan (2011) apontam soluções como períodos de pausa e reposição hídrica a fim de diminuir o tempo de exposição do trabalhador ao ambiente, para que o organismo possa eliminar o excesso de carga térmica e restabelecer seu equilíbrio.

A eficiência dos mecanismos termorreguladores às altas temperaturas ambientais e à umidade do ar, juntamente com o trabalho muscular, leva o trabalhador ao estresse por calor (SALAVISA, 2012). Neste contexto, o conforto térmico pode influenciar os fisioterapeutas e funcionários do setor de fisioterapia aquática da $\mathrm{AACD}$, mas como medida preventiva são constantemente orientados a realizar períodos de repouso para restabelecer o equilíbrio térmico (períodos de pausa a cada três horas trabalhadas) e reposição hídrica.

A resposta ao aumento da temperatura é a vasodilatação cutânea, onde a pele fica aquecida para eliminar o calor do corpo por evaporação de água. Quando a água se evapora, o indivíduo pode apresentar cansaço, sonolência, redução do desempenho físico e aumento de erros devido à perda de atenção e concentração. Com efeito, a reposição hídrica é fundamental para restabelecer a homeostase térmica (KROEMER; GRANDJEAN, 2005; SALAVISA, 2012).

Conforto acústico é a inteligibilidade das palavras e privacidade da fala, proporcionando um ambiente agradável de trabalho. O fato deve ser considerado em ambiente terapêutico (AZEVEDO, 2007). Com relação ao conforto acústico no ambiente deste estudo, pode-se dizer que é poluído, pois os terapeutas compartilham o ambiente com outros terapeutas, incluindo pacientes adultos e infantis e seus acompanhantes. $\mathrm{Na}$ abordagem dos pacientes, muitas vezes, as crianças necessitam de canções lúdicas como estímulo na terapia e os adultos necessitam de ambiente calmo e tranquilo.

Os terapeutas oferecem diversos comandos verbais de acordo com o foco da terapia influenciando no conforto acústico do local deste estudo e, apesar da baixa potência da voz humana que pode chegar a 50 microwatt a uma distância de 11 metros, esta se dissipa quando o ambiente é amplo ou existem fatores correlacionados como o tráfego urbano e outros, como aparelhos sonoros (AZEVEDO, 2007).

Em relação ao ambiente lumínico, deve-se ficar atento para o excesso de iluminação, pois pode causar reflexos brilhantes dificultando a visibilidade e a segurança no local de trabalho (AZEVEDO, 2007; SKINNER; THOMSON, 1985; COLE; MORRIS; RUOTI, 2000). No ambiente de trabalho desta pesquisa a iluminação está direcionada ao redor da piscina, fato esse que não interferiu no estudo.

Uma preocupação importante na atividade de reabilitação na piscina são os cuidados com a água, pois esta se não bem controlada, poderá oferecer riscos para o fisioterapeuta e o paciente. Os estudos de Campo e Quiroz (2003), Guedes (2013) e Azevedo (2007) apontam que a qualidade físicoquímica e microbiológica da água deve ser primordial, e esta deve estar isenta de microorganismos patogênicos capazes de alterar a saúde. Além disso, é preciso controlar os parâmetros físico-químicos que possam provocar qualquer tipo de distúrbio ou doença, pois os terapeutas e os funcionários se expõem constantemente no ambiente de trabalho.

A faceta dor e desconforto, descrita no Domínio Físico apresentou uma relevância significativa. Trata-se de fator relevante, pois os terapeutas e funcionários têm foco principal na reabilitação de pacientes neurológicos, e estes pacientes demandam posições desconfortáveis para a realização das atividades propostas e podem influenciar na capacidade musculoesquelética do fisioterapeuta. Estes dados corroboram com estudo de Pivetta, Jacques e Lopes (2005) que relata a presença desses distúrbios em fisioterapeutas e funcionários. Descrevem que as especialidades clínicas de área de atuação ortopédica, traumatológica, neurológica e cardiológica são as áreas mais frequentes de alterações musculoesqueléticas. Já as áreas com menor frequência de lesões são: 
dermato-funcional, oncologia, neonatologia, ginecologia e obstetrícia, preventiva, fisioterapia geral e hidroterapia (ROMANI, 2001; CIARLINI; MONTEIRO; BRAGA, 2004).

A lombalgia em fisioterapeutas é o principal sintoma de dor, sendo relacionada às sobrecargas físicas e apresentando o acometimento precoce de forma frequente. A dor lombar interfere diretamente em seu trabalho e em sua vida particular, indicando a importância de ações preventivas desde o início da prática profissional (ARANTES; NUNES; PERNAMBUCO, 2009). Já os funcionários apresentam sobrecarga biomecânica, pois são responsáveis pela transferência dos pacientes para piscina e auxiliam no vestuário dos mesmos. Pensando nesses aspectos, os funcionários são treinados constantemente, orientados para evitar algias e desempenhar a função com maior segurança.

Entre as medidas preventivas em relação aos distúrbios musculoesqueléticos faz-se necessário algumas medidas como: prática regular de atividade física, adaptação ergonômica do local de trabalho, orientações especialmente de movimentos e posturas de risco para o desenvolvimento de distúrbios musculoesquelético (BAGALHI; ALQUALO-COSTA, 2011).

Como visto, muitos são os fatores que interferem na qualidade de vida dos profissionais avaliados. Neste estudo verificou-se que a qualidade de vida dos fisioterapeutas e funcionários que trabalham no setor de fisioterapia aquática da AACD sofre alteração pelo ambiente físico e aspectos psicológicos. Sugere-se para futuros estudos aumentar o tamanho da amostra e comparar com outros fisioterapeutas e colaboradores de centros de fisioterapia aquática.

\section{Referências}

AMORIM, C. Síndrome de Burnout em fisioterapeutas e acadêmicos de fisioterapia: um estudo preliminar. In: BENEVIDES, P. Burnout: quando o trabalho ameaça o bem estar do trabalhador. São Paulo: Casa do Psicólogo, 2002.

ARAÚJO, T. C. C. F. Comunicação em saúde: contribuições do enfoque observacional para pesquisa e atuação. Arquivos Brasileiros de Psicologia, v. 61, n. 2, p. 1-13, ago. 2009.

ARANTES, A. S.; NUNES, A. P. R. O.; PERNAMBUCO, A. P. Prevalência de lombalgia nos fisioterapeutas atuantes na cidade de Formiga-MG. Conexão ciência, v. 4, n. 1; p. 44-52. 2009.

AZEVEDO, M. B. Compatibilização do conforto acústico com o conforto térmico no ambiente construído centro integrado de educação pública. 2007. 120 f. Dissertação (Mestrado em Arquitetura) - Programa de Pós-Graduação em Arquitetura, Universidade Federal do Rio de Janeiro, Rio de Janeiro.

BAGALHI, C. T.; ALQUALO-COSTA, R. Prevalência de distúrbios osteomusculares relacionados ao trabalho em fisioterapeutas. Science in Health, v. 2, n. 2, p. 93-102, maio/ago. 2011.

BATISTA, D. A. O ser fisioterapeuta: desenvolvimento profissional e qualidade de vida no trabalho. 2010. 127 f. Dissertação (Mestrado em Desenvolvimento Regional) - Programa de PósGraduação em Gestão Estratégica de Empreendimentos. Faculdades ALFA, Goiânia.

BARROS, F. B. M. Poliomielite, filantropia e fisioterapia: o nascimento da profissão de fisioterapeuta no Rio de Janeiro dos anos 1950. Ciência e Saúde Coletiva, v. 13, n. 3, p. 941-954, maio/jun. 2008. crossef

BENEVIDES-PEREIRA, A. M. T. Burnout: quando o trabalho ameaça o bem estar do trabalhador. São Paulo: Casa do Psicólogo, 2002. 
CAMARGO, M. G.; FURLAN, M. M. D. P. Resposta fisiológica do corpo às temperaturas elevadas: exercício, extremos de temperatura. Revista Saúde e Pesquisa, Maringá, v. 4, n. 2, p. 278-288, maio/ago. 2011.

CAMPO, N. C. del; QUIROZ, C. C. Riesgos microbiológicos associados al uso de piscinas públicas. Água Latinoamérica, p. 16-20, mar/abr. 2003. Disponível em: http://www.agualatinoamerica.com/docs/pdf/nivel\%20basico\%20mar03.pdf. Acesso em: 26 jan. 2014.

CANIZARES, J. Como implementar ações de qualidade de vida em empresas. 2008. Disponível em: $\quad$ http://www.administradores.com.br/artigos/administracao-e-negocios/como-implementaracoes-de-qualidade-de-vida-em-empresas/20867/. Acesso em: 15 maio 2013.

CARVAlHO, L.; MALAGRIS, L. E. Avaliação do nível de stress em profissionais de saúde: Estudos e Pesquisas em Psicologia, v. 7, n. 3, p. 210-222, 2007.

CIARLINI, I. de A.; MONTEIRO P. P.; BRAGA R. O. M. Lesões por esforços repetitivos em fisioterapeutas. Revista Brasileira em Promoção da Saúde, Fortaleza, v. 18, n. 1, p. 11-16, 2004.

COLE, A. J.; MORRIS, D. M.; RUOTI, R. G. Reabilitação aquática. São Paulo: Manole, 2000.

COSTA, R. P. Interdisciplinaridade e equipes de saúde: concepções. Mental, v. 5, n. 8, p. 107-124, jun. 2007.

COSTA, R. K. S.; ENDERS, B. C.; MENEZES, R. M. P. Trabalho em equipe em saúde: uma análise contextual. Ciência, Cuidado e Saúde, v. 7, n. 8, p. 530-536, out./dez. 2008.

FLECK, M. P. de A. O instrumento de avaliação de qualidade de vida da Organização Mundial da Saúde (WHOQOL-100): características e perspectivas. Ciência e Saúde Coletiva, v. 5, n. 1, p. 3338, 2000. rossef

GUEDES, A. S. Epidemiological barriers of aquatic therapy pools. The Health, v. 4, n. 2, p. 24-29, 2013.

KROEMER, K. H. E.; GRANDJEAN, E. Manual de ergonomia: adaptando o trabalho ao homem. 5. ed. Porto Alegre: Bookman, 2005.

LACAZ, F. A. de C. Qualidade de vida no trabalho e saúde/doença. Ciência \& Saúde Coletiva, v. 5, n. 1, p. 151-161, 2000. rossef

MAIA, I. M. O.; FRANCISCO, A. C. de. Ergonomia: ferramenta de manutenção industrial. In: CONGRESSO INTERNACIONAL DE ADMINISTRAÇÃO, 2007, Ponta Grossa. Anais... Ponta Grossa: UEPG, 2007.

MINAYO, M. C. de S. Violência Social sob a perspectiva da saúde pública. Caderno de Saúde Pública, v. 10, n. 1, p. 7-18, 1994.

MINAYO, M. C. de S.; HARTZ, Z. M. de A.; BUSS, P. B. Qualidade de vida e saúde: um debate necessário. Ciência da Saúde Coletiva, Rio de Janeiro, v. 5, n. 1, p. 7-18. 2000.

NUNES, S, F. P.; NASSARALLA, I. Pedagogia institucional: fatores humanos nas organizações. Rio de Janeiro: ZIT Editores, 2004. 
PIVETTA, A. D.; JACQUES, M. A.; LOPES, L. F. Prevalência de distúrbios osteomusculares relacionados ao trabalho em fisioterapeutas. Efdeportes, Buenos Aires, v. 10, n. 80, 2005. Disponível em: http://www.efdeportes.com/efd80/dort.htm. Acesso em: 12 mar. 2013.

RIBEIRO, E. M. A qualidade de vida na estratégia de saúde da família: refletindo sobre significados. Revista Família Saúde e Desenvolvimento, v. 3, n. 2, p. 109-115, jul./dez. 2001.

ROMANI, J. C. P. Distúrbios musculoesqueléticos em fisioterapeutas: incidência, causas e alterações na rotina de trabalho. 2001. 107 f. Dissertação (Mestrado em Engenharia de Produção) Universidade Federal de Santa Catarina, Florianópolis.

SALAVISA, A. Contributo para o levantamento da realidade dos fisioterapeutas portugueses que trabalham em meio aquático - Segurança, Nível de Treino e Responsabilidades. $2012.149 \mathrm{f}$. Dissertação (Mestrado em Fisioterapia) - Programa de Licenciatura em Fisioterapia, Faculdades Barcarena, Portugal.

SEIDL, E. M. F.; ZANNON, C. M. L. C. Qualidade de vida e saúde: aspectos conceituais e metodológicos. Caderno de Saúde Pública, v. 20, n. 2, p. 580-588, 2004. Crossef

SILVA, J. B.; BRANCO, F. Fisioterapia aquática funcional. São Paulo: Artes Médicas, 2011.

SKINNER, A. T.; THOMSON, A. M. Duffield: exercícios na água. 3. ed. São Paulo: Manole, 1985.

VILLAROUCO, V.; ANDRETO, L. F. M. Avaliando desempenho de espaços de trabalho sob o enfoque da ergonomia do ambiente construído. Produção, v. 18, n. 3, p. 523-539, set./dez. 2008. crossef 